\title{
Análisis de los niveles de burnout en deportistas en función del nivel de inteligencia emocional percibida: el papel moderador de la personalidad
}

\author{
Analysis of burnout's levels in athletes in function of emotional \\ intelligence perceived: role moderator of personality
}

\section{A análise dos níveis burnout's em atletas em função da inteligência emocional percebida: o papel moderador da personalidade}

\author{
J. González ${ }^{1}$, A.B. Ros ${ }^{2}$, M.I. Jiménez ${ }^{2}$ y E. Garcés de los Fayos ${ }^{1}$ \\ ${ }^{1}$ Universidad de Murcia (España), ${ }^{2}$ Universidad Católica San Antonio, Murcia (España)
}

Resumen: El constructo psicológico "Inteligencia Emocional” (IE) hace referencia a la capacidad de las personas para integrar eficazmente aspectos cognitivos y emocionales. La personalidad, entendida como un sistema psicológico que emerge de la interacción-adaptación entre individuo y ambiente, media entre el funcionamiento intrapsíquico (autopercepción, autoestima, autoconocimiento y autorregulación) y evoluciona permanentemente con el medio. Por otro lado, la consolidación teórica del burnout en el ámbito de la psicología del deporte, es cada vez más evidente. Por ello, y ante la escasez de trabajos dirigidos a analizar la existencia de relación entre constructos, el objetivo del presente trabajo ha consistido en comprobar si los niveles de Inteligencia Emocional Percibida (IEP) en una muestra de 201 deportistas, se relaciona con sus niveles de burnout, considerando el papel moderador que desempeńa la personalidad. Se han utilizado el Trait Meta-Mood Scale (TMMS-24) para la medida de la IE, el NEO-FFI para la medida de la personalidad, y el IBD-R para la medición de burnout. Los resultados obtenidos en nuestro trabajo muestran que los factores neuroticismo y responsabilidad, moderan la relación existente entre atención emocional y el agotamiento emocional.

Palabras Clave: Atención emocional, agotamiento emocional, neuroticismo, responsabilidad, deportistas.

Abstract: Emotional Intelligence (IE) is a psychological construct that refers to the individuals' capacity to integrate cognitive and emotional aspects effectively. Personality could be understood as a psychological system that emerges from the interaction between individual and environment, which evolves permanently mediating the intrapersonal functioning (such as self-perception, self-esteem, self-knowledge and self-regulation) and personal adaptation. On the other hand, the theoretical consolidation of the burnout in sport's psychology field is increasing. However, there is still relatively scarce investigation about the relationship between is study aims at analyzing the existence of the relation between levels of Perceived Emotional Intelligence (PEI) and burnout's symptoms, considering the moderator role of personality. Participants were 201 athletes that completed the TMMS-24 for the measurement of PEI, the Neo FFI for personality assessment, and IBD-R to establish burnout's levels. The results of this research suggest that the relationship between emotional attention and emotional exhaustion is mediated by neuroticism and conscientiousness factors. Keywords: Emotional attention, emotional exhaustion, neuroticism, conscientiousness, athletes.

Resumo: A construção psicológica "Inteligência Emocional" (EI) refere-se à capacidade das pessoas de integrar efetivamente os aspectos cognitivos e emocionais. Personalidade, entendido como um sistema psicológico que emerge da interação entre adaptação individual e meio ambiente, faz a mediação entre o intrapsíquico funcionamento ( auto- percepção, auto-estima, auto-conhecimento e auto-regulação) e em constante evolução médio. Além disso, a consolidação teórica do burnout no campo da psicologia do esporte, é cada vez mais evidente. Por isso, e devido à falta de estudos que visam analisar a existência de relação entre os construtos, o objetivo deste trabalho foi testar se os níveis de Inteligência Emocional Percebida (IEP), em uma amostra de 201 atletas, está relacionado com os seus níveis de Burnout, considerando o papel moderador da personalidade. Usamos o Trait Meta- Mood Scale (TMMS -24) para a medição de EI, o NeoFFI para medir a personalidade, e IBD -R para medir burnout. Os resultados do nosso estudo mostram que fatores de neuroticismo e responsabilidade, moderou a relação entre o cuidado emocional e exaustão emocional.

Palavras-chave: atenção emocional, exaustâo emocional, neuroticismo, consciência, atletas.

\section{Introducción}

El concepto Inteligencia Emocional (IE) hace referencia a la capacidad de las personas para integrar eficazmente aspectos emocionales y cognitivos (Salovey y Grewal, 2005).

Las investigaciones realizadas en los últimos años han evidenciado que ser emocionalmente inteligente supone im-

Dirección para correspondencia [Correspodence address]: Juan González Hernandez. Universidad de Murcia. E-mail: jgonzalez@um.es portantes beneficios en diferentes áreas del funcionamiento humano (Extremera, Fernández-Berrocal y Salovey, 2006; Martins, Ramalho y Morin, 2010), estableciendo relaciones positivas y significativas entre la IE e indicadores de comportamiento adaptado (Palomera, Fernández-Berrocal y Barckett, 2008; Zeidner, Roberts y Matthews, 2008), relaciones interpersonales y apoyo social (Jiménez y López-Zafra, 2011), me- 
nos tendencia a presentar comportamientos disruptivos y agresivos (Extremera y Fernández-Berrocal, 2004; Garaigordobil y Oñederra, 2010; Petrides, Frederickson y Furham, 2004), y el empleo de estrategias de afrontamiento efectivas situaciones de estrés (Extremera, y Fernández-Berrocal, 2006; Pérez y Castejón, 2007).

De la misma forma, la IE actúa como un factor protector, que se relaciona con mayores niveles de bienestar emocional y ajuste psicológico (Salovey, Stroud, Woolery y Epel, 2002; Ciarrochi, Forgas, y Mayer, 2001, 2006; Ramos, Fernández-Berrocal y Extremera, 2007), una mayor salud mental (Fernández-Berrocal, Alcaide, Extremera y Pizarro, 2006; Martins, Ramalho y Morin, 2010; Salguero, Palomera y Fernández-Berrocal, 2012) y, en definitiva, niveles superiores de satisfacción vital (Extremera y Fernández-Berrocal, 2005; Furnham y Petrides, 2003).

A nivel teórico, se distinguen dos aproximaciones que engloban conceptualizaciones de las que se derivan dos tipos de modelos teóricos que podemos considerar complementarios en cuanto al estudio de la IE. Por un lado, se encuentran los modelos basados en el procesamiento de información emocional centrado en las habilidades emocionales básicas (Mayer y Salovey, 2007), que estudian habilidades concretas como identificar emociones en caras, o comprensión de significados emocionales (Mayer, Roberts y Barsade, 2008); y por otro, aquellos modelos denominados "mixtos" (Bar-On, 2006; Goleman, 2005, Pena y Repetto, 2008), cuya visión es más global, que se centran en rasgos estables de comportamiento y en variables de personalidad (empatía, asertividad, impulsividad, etc.) (Fernández-Berrocal y Ruíz, 2008; Mayer, Salovey y Caruso 2000).

Con respecto al segundo tipo de modelos, se consideran "mixtos" al combinar dimensiones de tipo cognitivo, social, emocional y de personalidad, destacando el Modelo de Inteligencia Emocional-Social de Bar-On (2006) que concibe la inteligencia emocional-social como una intersección de competencias sociales y emocionales, de destrezas y de facilitadores que interactúan entre sí y que determinan la efectividad con la que afrontamos las demandas diariamente (Bar-On, 2000).

Según este planteamiento, el constructo inteligencia emocional-social (ESI), está formado por una compleja interacción de rasgos emocionales y de personalidad que conforman al individuo. Por ello, definió en términos darwinianos el resultado de la conducta emocional y socialmente inteligente, planteando que las personas emocionalmente inteligentes son adaptativamente optimistas, flexibles, realistas, resuelven los problemas eficazmente, afrontando el estrés sin perder el control (Bar-On, 2006; Reiff, Hatzes, Bramel y Gibbon, 2001).
Figura 1. Modelo ESI de Bar-On

\begin{tabular}{ll}
\hline Factor & Subfactores \\
\hline Destreza Intrapersonal & Autorrefuerzo \\
& Autoconciencia Emocional \\
& Asertividad \\
& Independencia \\
& Auto-actualización \\
\hline Destreza Interpersonal & Responsabilidad Social \\
& Empatía \\
& Relaciones Interpersonales \\
\hline Adaptabilidad & Evaluación de la realidad \\
& Flexibilidad \\
& Solución de problemas \\
\hline Control del estrés & Tolerancia a la frustración \\
& Control de impulsos \\
\hline Humor General & Optimismo \\
& Felicidad \\
\hline
\end{tabular}

Las diferencias individuales señalan la forma de interpretación psicológica y reactividad de la respuesta ante las circunstancias, atendiendo a los elementos que aportan la experiencia adquirida y los más asociados al carácter del individuo. Por ello, la idea que las características de personalidad desempeñan una notable influencia sobre la forma de afrontar el estrés, los niveles de bienestar y conductas preventivas, constituye un área de gran interés en la que han venido a converger numerosas investigaciones (Bermúdez, 1999; DeNeve y Cooper, 1998; Smith y Christensen, 1995; Van Heck, 1997).

Entendiendo que la personalidad se construye psicosociológicamente, podrían explicarse la definición de un estilo de vida determinado mucho más de lo que lo hacen las situaciones o rasgos por sí solos, permitiendo indagar cómo las personas que realizan cualquier tipo de actividad se adaptan individualmente en contextos concretos (González, Garcés de los Gayos y Ortega, 2014; Hopkins, 2005; Paton y Hopkins, 2005).

En este sentido, el deporte se convierte en un contexto interesante de estudio, ante su variabilidad y búsqueda de la adaptación ante objetivos constantemente cambiantes (Hansen, Larson y Dworkin, 2003).

Por ello, no es sorprendente el creciente interés de los investigadores por analizar la utilidad de la inteligencia emocional en el contexto deportivo (Meyer y Fletcher, 2007; Ros, Moya y Garcés de los Fayos, 2013). En los últimos años se han generado líneas de investigación muy dispares y se han publicado diferentes estudios en este ámbito, en los que se ha relacionado la inteligencia emocional, con variables tales como el rendimiento (Botterill y Brown, 2002) o la ansiedad precompetitiva (Lane, Thelwell y Devonport, 2009). 
El burnout en el deporte y la actividad física es un fenónemo multidimensional y multicausal, que como síndrome psicológico se caracteriza fundamentalmente por el cansancio emocional y físico, la disminución de logros y la devaluación deportiva. Aunque existen diferentes modelos teóricos explicativos del burnout, a todos subyace como denominador común, el estrés como elemento causal del mismo, generado como consecuencia de un ineficaz proceso de adaptación del deportista ante situaciones de sobreentranamiento, sobreexigencia, estancamiento o el retiro de la carrera "(Cox, 2009; Garces y Vives, 2003).

El estudio del burnout entiende que no todas las variables personales ejercen la misma influencia en todo el proceso de "desgaste individual" (Augusto-Landa, Berrios-Martos, López-Zafra y Aguilar, 2006; Moreno, Rodriguez, Garosa y Morante, 2005). En el mundo del deporte, los estudios de variables de la personalidad y su relación con el burnout deben centrarse en el descubrimiento de elementos que faciliten la focalización en recursos personales, aportando así formas diferentes de afrontar situaciones, que pueden desembocar en un determinado desgaste emocional. En este sentido, cada vez es mayor la evidencia empírica que muestra la relación existente entre la personalidad y el burnout (Alarcon, Eschleman y Bowling, 2009).

Entre las variables que se han planteado como potenciales predictoras del síndrome (Garcés de Los Fayos y Vives, 2003), se encuentra un estilo negativo de dirección y de interacción por parte del entrenador, altas demandas competitivas, excesivas presionestemporales, monotonía del entrenamiento (sensación de aburrimiento y falta de estímulo), sentimientos de aislamientoy falta de apoyo en su grupo de referencia, carencia de refuerzos positivos, déficit de habilidades o, estrategias de afrontamiento ineficaces, frustración cuando los logros obtenidos no se corresponden con las expectativas iniciales, , intereses mercenarios de los padres y estilo de vida externo no apropiado con el rigor exigido al deportista (Carlin y Garcés de los Fayos, 2010).

El objetivo del presente estudio, sustentado científicamente en el modelo de Inteligencia Social-Emocional de Bar-On (2006), ha consistido en comprobar si los niveles de Inteligencia Emocional Percibida (IEP) de una muestra de deportistas, se relacionan con sus niveles de burnout, contemplando la influencia moderadora que ejercen las diferencias de personalidad en la citada relación, teniendo en cuenta las diferencias de género.

\section{Metodo}

Se presenta un estudio no experimental, predictivo en una muestra de deportistas, atendiendo a sus condiciones de género y tipo de deporte que realizan, diferenciando entre colectivos e individuales.

\section{Participantes}

Se dispone de una muestra aleatoriamente distribuida de deportistas federados $(N=201)$, con edades comprendidas entre 18 y 35 años, con una media de edad de 20,87 ( $D T=2,76)$, que compíten en alguna modalidad deportiva (fútbol, balonmano, tenis, atletismo y deporte de vela). De la misma, un $83,3 \%$ son hombres $(n=168)$ y un $16,7 \%(n=33)$ son mujeres, así como un $38,8 \%$ practica deportes individuales $(n=78)$ y un $51,2 \%$ deportes colectivos $(n=103)$, con una media de práctica semanal de 6,28 horas (DT $=4,316)$.

\section{Instrumentos}

Inteligencia emocional. Para la evaluación de la Inteligencia Emocional Percibida (IEP), se empleó la versión española del Trait Meta-Mood Scale (TMMS-24; Fernández-Berrocal, Extremera y Ramos, 2004) que contiene 24 items, a través de una escala tipo Likert de 5 puntos. Proporciona la estimación del propio sujeto sobre su su capacidad para manejar emociones y sentimientos. La escala contiene tres factores o subescalas que proporcionan tres puntuciones: Atención a los sentimientos (capacidad para monitorizar sentimientos y emociones), e.g., "Normalmente me preocupo mucho por lo que siento"; Claridad emocional (habilidad para distinguir emociones y sentimientos), e.g., "A menudo me doy cuenta de mis sentimientos en diferentes situaciones"; y Reparación emocional (habilidad para regular estados displacenteros o mantener estados afectivos positivos), e.g., "Intento tener pensamientos positivos, aunque me sienta mal". La fiabilidad interna mostrada por cada uno de los componentes es Atención $\alpha=, 79$, Claridad $\alpha=, 83, y$ Reparación $\alpha=, 86$.

Burnout. El Inventario de Burnout en Deportistas- Revisado (IBD-R; Garcés de Los Fayos, De Francisco y Arce, 2012) es la readaptación del Inventario de Burnout en Deportistas (IBD) de Garcés de Los Fayos (1999) formado por 26 ítems para la medida de las tres dimensiones del burnout propuestas por Maslach y Jackson (1981): agotamiento emocional, reducida realización personal y despersonalización. El formato de respuesta fue tipo Likert, con cinco alternativas: Nunca he sentido o pensado esto (1), Alguna vez al año lo he pensado o sentido (2), Alguna vez al mes lo he pensado o sentido (3), Alguna vez a la semana lo he pensado o sentido (4), Pienso $o$ siento esto a diario (5). El IBD-R cuenta con los tres factores asociados a las dimensiones explicativas del síndrome, si bien se ha reducido el mismo a un total de 19 ítems. Con adecuados valores de los índices de ajuste $\left(\mathrm{c}^{2} / \mathrm{gl}=2,04\right.$, RMSEA= ,05, $\mathrm{SRMR}=, 06, \mathrm{NNFI}=$,93, CFI= ,94 y GFI= ,92), y por lo que se refiere a la consistencia interna para cada uno de los 19 ítems, este modelo más parsimonioso en ítems sigue mostrando valores de Alpha de Cronbach mayores o iguales a ,70. Dicha consistencia por dimensiones fue de $\alpha=, 72$ para 
Despersonalización, $\alpha=70$ para Reducida realización personal, y $\alpha=, 73$ para Agotamiento emocional.

Personalidad. Para la evaluación de la personalidad se ha empleado el Inventario de Evaluación de la Personalidad normal NEO-FFI abreviado ( Costa, P. y McRae, R., 1989, adaptación al castellano realizada por Sanz y cols., 1999); versión con 60 elementos, evalúa 5 factores de personalidad: El factor Neuroticismo (N), que contrapone el ajuste y la estabilidad emocional al desajustey la tendencia a estar ansioso, irritado, triste y afligido.; El factor Extraversión (E), valora la tendencia a la sociabilidad o la preferencia por estar con otras personas y vinculadoa diferentes grupos. Incluye facetas comola asertividad y la tendencia a tener un estado de ánimo alegre, enérgico y optimista, asi comola búsqueda de excitación y estimulación; El factor Apertura (O), que evalúa la tendencia a prestar atención a los sentimientos interiores, la preferencia por la variedad, la sensibilidad estética, la curiosidad intelectual y la independencia de juicio; El factor Amabilidad (A), valora las si las tendencias interpersonales se basan en la cooperación, el altruismo, la empatía o por el contrario, la persona muestra una actitud desconfiada y suspicaz con respecto a las intenciones de los demás.. Por último, el factor Responsabilidad (C), valora la capacidad de la persona para la planificación y ejecución de las tareas con voluntariedad y decisión, así como la motivación de logro y la autodisciplina que, generalmente, se asocian a un buen rendimiento académico o profesional. La fiabilidad interna mostrada por cada uno de los factores del inventario es de $\alpha=, 90$ para el factor Neuroticismo, de $\alpha=, 84$ para el factor Extraversión, de $\alpha=, 82$ para el factor Apertura, de $\alpha=, 83$ para el factor Amabilidad y, por último, de $\alpha=, 88$ para el factor Responsabilidad.

\section{Procedimiento}

La muestra se ha seleccionado aleatoriamentede entre un grupo de deportistas federados a los que se les realizó un llamamiento para la participación en el estudio. Los participantes del estudio han sido deportistas pertenecientes a diversas federaciones deportivas y que compiten en diferentes categorías de formación (senior, juveniles, y tecnificación). El protocolo de evaluación empleado para la realización de este estudio, se administró a los deportistas de forma colectiva.....antes de comenzar el entrenamiento

Los deportistas, cumplimentaron los cuestionarios, previa explicación de los objetivos del estudio y tras rellenar la autorización correspondiente mediante consentimiento informado.

\section{Análisis de datos}

Se realiza análisis descriptivo de tendencias centrales y dispersión (media, desviación típica y curtosis) y homegenidad de varianzas. Para la relación entre las variables objeto de estudio, se disponen pruebas t de diferencias muestrales y análisis de moderación (Hayes y Matthes (2009). Se utiliza el paquete estadístico SPSS, en su versión 20.0.

\section{Resultados}

Tras los análisis realizados, en la tabla 1 pueden apreciarse que cuando se comparan las muestras en relación a la variable género, las mujeres indican significativamente mayores niveles de neuroticismo $\left(t_{2,199}=4.224 ; p=, 041\right)$, y los hombres muestran niveles mayores en claridad emocional $\left(t_{2,199}=4.926 ; p=, 028\right)$. Aunque no significativa, si se aprecia una tendencia a favor de los hombres, en despersonalización $\left(t_{2,199}=3,040 ; p=, 083\right)$.

Tabla 1. Indicadores de tendencias centrales, dispersión, y diferencias según género y deporte

\begin{tabular}{|c|c|c|c|c|c|c|c|c|c|c|c|}
\hline & \multirow{2}{*}{\multicolumn{5}{|c|}{ Personalidad }} & \multirow{2}{*}{\multicolumn{3}{|c|}{ Inteligenica emocional }} & \multirow{2}{*}{\multicolumn{3}{|c|}{ Burnout }} \\
\hline & & & & & & & & & & & \\
\hline & $\begin{array}{l}\text { Neuro- } \\
\text { ticismo }\end{array}$ & $\begin{array}{l}\text { Extra- } \\
\text { version }\end{array}$ & Apertura & $\begin{array}{l}\text { Cordia- } \\
\text { lidad }\end{array}$ & $\begin{array}{l}\text { Responsa- } \\
\text { bilidad }\end{array}$ & Atención & Claridad & $\begin{array}{l}\text { Repara- } \\
\text { ción }\end{array}$ & $\begin{array}{l}\text { Agemo- } \\
\text { cional }\end{array}$ & $\begin{array}{l}\text { Des- } \\
\text { pers. }\end{array}$ & $\begin{array}{c}\text { R.R. } \\
\text { Personal }\end{array}$ \\
\hline Media (DT) & $\begin{array}{c}31,30 \\
(6,069)\end{array}$ & $\begin{array}{c}39,60 \\
(4,039)\end{array}$ & $\begin{array}{c}37,27 \\
(4,577)\end{array}$ & $\begin{array}{c}36,73 \\
(3,911)\end{array}$ & $\begin{array}{c}42,97 \\
(5,256)\end{array}$ & $\begin{array}{c}26,10 \\
(6,650)\end{array}$ & $\begin{array}{c}27,34 \\
(6,092)\end{array}$ & $\begin{array}{c}29,32 \\
(6,222)\end{array}$ & $\begin{array}{c}11,74 \\
(3,648)\end{array}$ & $\begin{array}{l}26,76 \\
(4384)\end{array}$ & $\begin{array}{c}9,69 \\
(3927)\end{array}$ \\
\hline Curtosis &,- 006 &, 321 & 1,157 &, 844 &,- 001 &,- 596 &,- 780 &,- 443 &,- 125 &, 151 & ,935 \\
\hline género $t_{199,2}$ & $\begin{array}{r}-4,224 \\
(, 041)\end{array}$ & $\begin{array}{l}, 790 \\
(, 375)\end{array}$ & $\begin{array}{l}, 434 \\
(, 511)\end{array}$ & $\begin{array}{l}, 284 \\
(, 595)\end{array}$ & $\begin{array}{l}1,028 \\
(, 312)\end{array}$ & $\begin{array}{l}, 710 \\
(, 400)\end{array}$ & $\begin{array}{l}4,926 \\
(, 028)\end{array}$ & $\begin{array}{l}, 665 \\
(, 416)\end{array}$ & $\begin{array}{l}, 233 \\
(, 630)\end{array}$ & $\begin{array}{l}3,040 \\
(, 083)\end{array}$ & $\begin{array}{l}, 999 \\
(, 319)\end{array}$ \\
\hline Homogeneidad $\left(\chi^{2}\right)$ &,- 288 &, 110 &, 271 & ,978 &, 705 &, 559 &, 007 &, 807 &, 823 &, 003 & ,306 \\
\hline deporte $t_{199,2}$ & $\begin{array}{l}, 977 \\
(, 378)\end{array}$ & $\begin{array}{l}1,482 \\
(, 230)\end{array}$ & $\begin{array}{l}1,345 \\
(, 263)\end{array}$ & $\begin{array}{c}381 \\
(, 683)\end{array}$ & $\begin{array}{l}1,777 \\
(, 172)\end{array}$ & $\begin{array}{l}1,121 \\
(, 328)\end{array}$ & $\begin{array}{l}1,300 \\
(, 275)\end{array}$ & $\begin{array}{l}\text {,058 } \\
(, 944)\end{array}$ & $\begin{array}{l}1,081 \\
(, 341)\end{array}$ & $\begin{array}{l}, 097 \\
(, 908)\end{array}$ & $\begin{array}{l}1,019 \\
(, 363)\end{array}$ \\
\hline Homogeneidad $\left(\chi^{2}\right)$ &, 286 &, 119 & ,396 &, 744 &, 630 &, 495 &, 845 &, 706 &, 271 &, 225 &, 005 \\
\hline
\end{tabular}

Cuando se establecen indicadores de correlación bajos, aunque significativos entre las variables objeto de estudio (tabla
2), se observa la atención emocional aumenta conforme son más altos los niveles de neuroticismo $(r=, 160 ; p=, 024)$, aper- 
tura $(\mathrm{r}=, 174 \mathrm{p}=, 014)$ y cordialidad $(r=, 150 ; p=, 033)$. De la misma forma, los indicadores de reparación emocional son más altos conforme son más altos los niveles de apertura $(r=$ ,152; $p=, 032)$ y responsabilidad $(r=, 219 ; p=, 002)$. Se observa que la relación entre el agotamiento emocional y la extraver- sión es significativa y positiva $(r=, 131 ; p=, 049)$, de manera que conforme aumenta el agotamiento emocional, aparecen mayores indices de extraversión.

Tabla 2. Correlaciones entre las variables objeto de estudio

\begin{tabular}{llllll}
\hline & Neuroticismo & Extraversion & Apertura & Cordialidad & Responsabilidad \\
\hline Atención emocional &, $160^{*}(, 024)$ & &, $174^{*}(, 014)$ &, $150^{*}(, 033)$ & \\
Claridad emocional & & &, $152^{*}(, 032)$ & &, $219^{* *}(, 002)$ \\
Reparación emocional & & $.131^{*}(.049)$ & & \\
Agotamiento emocional & & & \\
Despersonalización & & & \\
Reducida realización & & & \\
$\mathrm{N}=201$ & & & \\
${ }^{*}$ es significativa de $\mathrm{p}<.05$ & & & \\
${ }^{* *}$ es significativa si $\mathrm{p}<.01$ & & &
\end{tabular}

Sin embargo, cuando se pretende establecer la fuerza moderadora de las características de personalidad sobre las relaciones predictivas, se observa que la atención emocional, predice negativa y significativamente el agotamiento emocional, cuando el neuroticismo es bajo $(p=, 0293$; interac $=, 0154)$, con una varianza explicada de un $65 \%\left(R^{2}=, 655\right)$ (ver figura 2$)$. De esta manera, cuando el neuroticismo es bajo, la vigilanciaatención de sus estados de ánimo en un esfuerzo por intentar comprenderlos, se convierte para los deportistas en una habilidad de inteligencia emocional que favorece menores niveles de agotamiento. Cuando el neuroticismo es medio o alto, la relación no es significativa.

Figura 2. Relación predictiva de la atención emocional sobre el agotamiento emocional con la moderación de neuroticismo

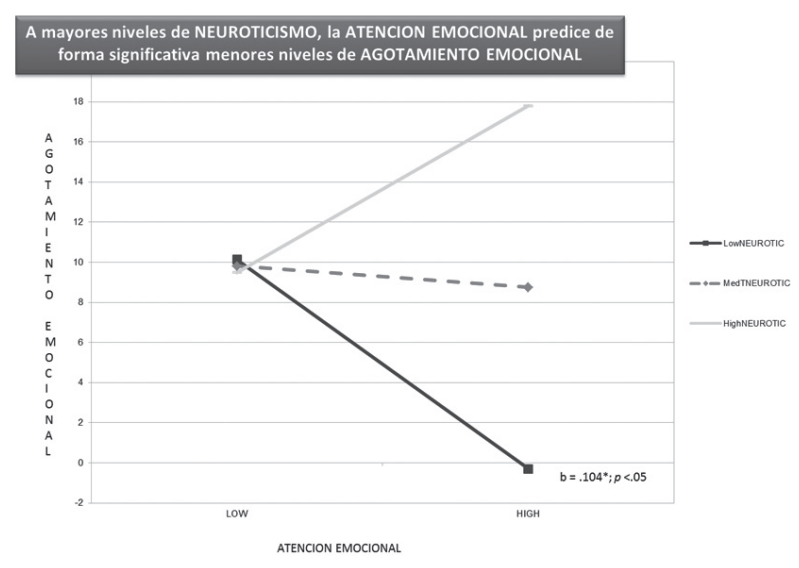

Se observa que la claridad emocional, predice positiva y significativamente el agotamiento emocional, cuando la respon- sabilidad es baja $(p=, 050$; interac $=-, 014)$, con una varianza explicada de un 60\% $\left(R^{2}=, 605\right)$ (ver figura 3). De esta manera, cuando la responsabilidad es baja, la claridad emocional se convierte en una habilidad de la inteligencia emocional que favorece el agotamiento. Por lo que con deportistas con pocos niveles de responsabilidad, trabajar en la identificación de emociones específicas atendiendo a sus reacciones, invirtiendo menos recursos cognitivos y así evaluar alternativas de acción, les supone mayor agotamiento emocional.

Figura 3. Relación predictiva de la claridad emocional sobre el agotamiento emocional con la moderación de responsabilidad

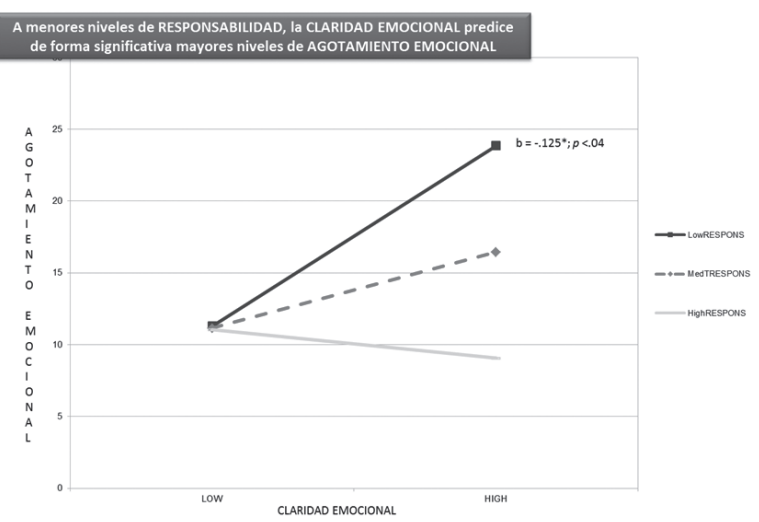

Se observa que la reparación emocional, predice negativa y significativamente el agotamiento emocional, cuando la responsabilidad es alta $(p=, 041$; interac $=-, 015)$, con una va-rianza explicada de un $63 \%\left(R^{2}=, 638\right)$ (ver figura 4$)$. De esta manera, cuando la responsabilidad es alta, la reparación 
emocional se convierte en una habilidad de la inteligencia emocional que permite disminuya el agotamiento. Por lo que con deportistas con alto nivel de responsabilidad, trabajar en la capacidad para regular estados emocionales, implicando la regulación de estados afectivos negativos, asi como la capacidad para prolongar estados afectivos positivos, supone proporcionarle herramientas que disminuyan la vulnerabilidad o la tendencia al agotamiento emocional.

Figura 4. Relación predictiva de la reparación emocional sobre el agotamiento emocional con la moderación de responsabilidad

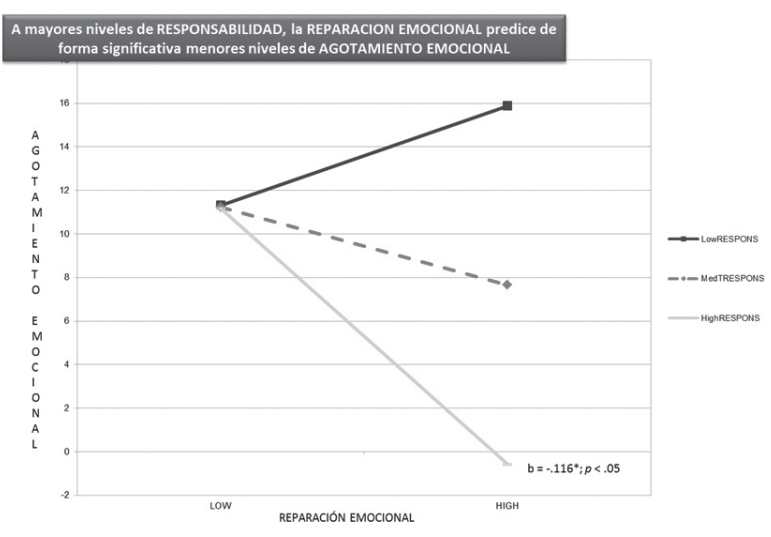

\section{Discusión}

Resultados como los del presente estudio, , muestran la necesidad de analizar tanto la prevalencia que el síndrome de burnout en los deportistas, como las variables antecedentes del problema (entre las que se encuentran las competencias sociales y emocionales), que pueden facilitar la aparición del burnout y de las consecuencias asociadas a este trastorno dentro del contexto deportivo (tales como problemas físicos, insatisfacción con el estilo de vida, problemas de motivación y de concentración, etc...) (De Francisco, Garcés de los Fayos y Arce, 2013).

Entre las variables predictoras del burnout, se encuentra el deterioro en las relaciones interpersonales, la falta de apoyo familiar y el desarrollo de una autoconfianza y autoconcepto más negativo (De Francisco et al., 2013), todas ellas relacionadas con habilidades de inteligencia emocional. Así, trabajos recientes de carácter clínico, han concluido que la IE es un constructo teóricamente relevante en la depresión, no solo por su relación con los estilos de afrontamiento, sino también porque reduce la vulnerabilidad a la emocionalidad negativa (ansiedad, enfado) (Salguero y Iruarrizaga, 2006), pudiendo predecir diferencias individuales en esta variable.

Los resultados obtenidos en diferentes investigaciones, llevan a pensar que en términos generales, que la atención emocional se relaciona negativamente con las vivencias de estrés y/o burnout (Extremera, Durán y Rey, 2007; Mikolajczak, Luminet, y Menil, 2006; Pena y Repetto, 2008). En general, el patrón característico de personas con mejores niveles de adaptación psicológica y menores niveles de burnout y estrés, han sido aquellas caracterizadas por puntuaciones moderadas-bajas en atención emocional y altas puntuaciones en claridad y reparación emocional (Extremera, Durán y Rey, 2009).

Los resultados encontrados en estudios con profesionales sanitarios, con una carga mental alta (enfermería), indican que una alta atención emocional se relaciona con altos niveles de estrés, menor satisfacción con lo que se hace y un bajo autoconcepto (Augusto-Landa, Berrios-Martos, López-Zafra y Aguilar, 2006; Augusto-Landa, López-Zafra, Aguilar-Luzón y Salguero de Ugarte, 2009).

El neuroticismo, como elemento de la personalidad que se desarrolla a través del proceso interactivo persona-deporte (González, Garcés de los Fayos y Ortega, 2014), incluye en sus facetas extremas la percepción de ansiedad, hostilidad, depresión, timidez, impulsividad, vulnerabilidad en condiciones de estrés -altos niveles-, así como equilibrio, pensamiento coherente, autocontrol, mayor capacidad comprensiva -bajos niveles-. De este modo, el neuroticismo, forma parte de un importante grupo de variables que influyen en el estado emocional, cognitivo y comportamental de los individuos (Lane, Devonport, Soos, Karsai, Leibinger y Hamar, 2010).

Es importante entender entonces, que los resultados obtenidos aportan la fuerza moderadora que la característica de personalidad está ejerciendo conforme las personas experimentan, ya que es en el proceso de aprendizaje donde la interacción facilita el autodescubrimiento de uno mismo y del manejo de situaciones sociales. En el caso del deporte, las situaciones combiantes que este plantea, principalmente en su expresión más competitiva, hacen que la persona conviva con estrés-control, con momentos de rapidez mental-control mental, euforia-decepción, entre otras, de forma constante.

Se ha evidenciado que elevadas puntuaciones en claridad y reparación emocional, se asocian con diferentes dimensiones de salud general y mayor adaptación a situaciones estresantes en el trabajo (Augusto-Landa et al. 2006), mayor satisfacción vital (Augusto-Landa et al. 2006), estrategias de afrontamiento positivas (Mikolajczak, Nelis, Hansenne y Quoidbach, 2008) y regulación emocional (Lane et al., 2010).

La mayoría de los estudios refleja la relación entre IE y personalidad, sobre todo cuando la IE se evalúa mediante pruebas de autoinforme (Mora y Martín, 2007; Petrides y Furnham, 2001). Estudios recientes (Extremera y FernándezBerrocal, 2005; Jiménez y López-Zafra, 2008), han mostrado la existencia de relaciones significativas entre las dimensiones de IE y los factores Neuroticismo y Extraversión. Los datos obtenidos en el presente estudio, complementan tales afirma- 
ciones, entendiendo que son en concreto el neuroticismo y la responsabilidad, los rasgos de la personalidad del deportista que moderan las relaciones entre las habilidades de IE y las respuestas de agotamiento emocional.

Precisamente, la inteligencia emocional entendida como un rasgo implica motivos (como la necesidad de rendimiento), estilos sociales (como la asertividad y la empatía), cualidades relacionadas con el self (como la autoestima), cualidades relacionadas con el control de la conducta (tales como la flexibilidad y el control de impulsos), cualidades emocionales (como la felicidad y el estado del animo) asi como el grado de autoconciencia emocional (Mayer, Roberts y Barsade, 2008).

Asimismo, es la condición responsable de la personalidad, la que se muestra más fuerte en su moderación sobre las relaciones entre claridad y reparación emocional y el agotamiento. Puede evidenciarse, que la relación entre las características de personalidad y las habilidades de IE es más fuerte que la que muestran con los indicadores de burnout; pero es cuando se establece la influencia de las primeras sobre las segundas, donde ya aparecen relaciones con las terceras.

Con respecto al factor neuroticismo, se establece de manera consistente, una relación negativa con rendimiento académico (Parker, Summerfeldt, Hogan y Majeski, 2004), autocontrol y perfecccionismo (Newsome, Day y Catano, 2000), y baja inteligencia emocional (Newsome et al., 2000). En este camino, los resultados obtenidos señalan que las relaciones entre la percepción de agotamiento y las dimensiones de atención, claridad y reparación emocional, exiten y son moderadas por las condiciones de personalidad.

Es la responsabilidad, el elemento diferencial de las relaciones entre claridad y reparación con el agotamiento emocional, lo que permite establecer que en base a los niveles de responsabilidad con los que se emprenden las tareas (en este caso, deportivas), la claridad emocional predice agotamiento (cuando la responsabilidad es baja), y la reparación emocional predice agotamiento emocional (cuando la responsabilidad es alta).

Por lo que respecta a las limitaciones de este estudio y planteamientos futuros de trabajo, se propone dirigir los esfuerzos investigadores hacia el logro de una fundamentación teórica más sólida del constructo Inteligencia Emocional rasgo dentro del ámbito deportivo.

Para ello, será necesario adaptar y/o crear diferentes instrumentos de evaluación de la IE que puedan ser aplicados específicamente en el contexto deportivo y que proporcionen información útil sobre los recursos emocionales de los que dispone el deportista y sobre su capacidad de autorregulación emocional en las diferentes condiciones que deberá afrontar en la situación deportiva. Para ello, y partiendo de postulados teóricos ya existentes, que aunque han surgido en otros contextos, cuentan con un apoyo empírico amplio, de manera que nos permitan comparar los resultados obtenidos en muestras de deportistas con los obtenidos en población general.

Este procedimiento, garantiza mayor profundización en las características diferenciales que presentan los deportistas, principalmente en situaciones de aprendizaje deportivo (indistintamente del nivel de práctica que realicen), así como en el conocimiento de la relación existente entre la personalidad y la capacidad deportiva.

La investigación en Psicología como ciencia, debe comprometerse a nivel de investigación, con el desarrollo de conocimientos y estrategias que procuren el bienestar personal, emocional y social de los deportistas en los distintos contextos en lo que se desenvuelven, y al mismo tiempo establecer relaciones con rendimientos personales y deportivos.

Desde el punto de vista más aplicado, este objetivo indefectiblemente requiere mejorar la calidad metodológica de los estudios, sin demorar el desarrollo de programas de prevención del burnout y de intervención, basados en la inteligencia emocional, que demuestren su eficacia para mejorar la capacidad deportiva con absoluta confianza y garantía.

\section{Aplicaciones prácticas}

El verdadero riesgo de las personas en general, y de los deportistas en particular, que atienden excesivamente a sus emociones sin la claridad y reparación adecuada, es que podrían desarrollar una espiral emocional que generase en un proceso rumiativo, fuera del control del sujeto, que más que aliviar su estado de ánimo perpetuará un estado de ánimo negativo, o lo que todavía es peor, desarrollar procesos negativos de autoconcepto o autoestima.

Por ello, al entrenar la claridad emocional, los deportistas identifican más fácilmente sus emociones específicas durante situaciones de estrés, pasarán menos tiempo atendiendo a sus reacciones emocionales, invirtiendo además menos recursos cognitivos, lo cual les permitirá evaluar alternativas de acción, mantener sus pensamientos en otras tareas o llevar a cabo estrategias de afrontamiento más adaptativas.

La reparacion emocional incide en que aquellos deportistas que son capaces de regular sus estados emocionales (interrumpir estados emocionales negativos y prolongar los positivos), son los que exhiben mejores niveles de rendimiento, por lo que en los planes de entrenamiento psicológico con los deportistas, necesitan introducirse estrategias que conlleven herramientas para el afrontamiento emocional adecuado de las situaciones.

El trabajo de entrenamiento psicológico con aquellos deportistas con alta inestabilidad emocional, presenta problemas a la hora de comprender y regular los estados emocionales negativos que tienden a experimentar. Por ello, el control emocional se convierte en un objetivo como tal dentro del entrenamiento para deportistas con el citado perfil, y no una 
condición asumida por entender que "el deportista ya lo ha experimentado con anterioridad".

Por el contrario los sujetos que puntúan bajo en neuroticismo -emocionalmente más estables-, muestran bajas puntuaciones en atencion y altas en comprensión y regulación, más identificado con quienes no están excesivamente pendientes de cómo se sienten, más capaces de identificar con claridad sus sentimientos y de disponer de habilidades y estrategias necesarias para poder controlar y regular sus emociones. Por ello, entrenar las habilidades de autocontrol permititrá a los deportistas compensar y de forma adaptativa, mejorar esos niveles neuróticos a través de la experiencias vividas, de las circunstancias de éxito o fracaso, de acercamiento o alejamiento con sus objetivos.
De todos modos, parece que es la responsabilidad, el factor que ejerce mayor influencia moderadora en la relación entre las habilidades emocionales y las respuestas de agotamiento, lo que implica que el entrenamiento específico en habilidades de perseverancia (focalización de objetivos realistas, estilo atribucional interno, conexión con el bienestar psicológico y utilidad percibida,entre otras) y la ordenación mental (respetar una temporalidad, flexibilizar los procesos de mejora, aceptar interrupciones en la consecución de logros, entre otras), podría facilitar que el deportista adquiera las habilidades, recursos y estrategias necesarias para afrontar eficazmente las condiciones cambiantes de la situación deportiva.

\section{Referencias}

1. Alarcon, G., Eschleman, K y Bowling, N. (2009). Relationships between personality variables and burnout: A meta-analysis. Work \& Stres, 23 (3), 244-263.

2. Augusto-Landa, J. M., Berrios-Martos, M. P., López-Zafra, E., y Aguilar, M. C. (2006). Relación entre burnout e inteligencia emocional y su impacto en la salud mental, bienestar y satisfacción laboral en profesionales de enfermería. Ansiedad y Estrés, 12 (2-3), 479-493.

3. Augusto-Landa, J. López-Zafra, E., Mtz. de Antoñana, R., y Pulido, M. (2006). Perceived emotional intelligence and life satisfaction among university teachers. Psicothema, 18, 152-157.

4. Augusto-Landa, J. M., López-Zafra, E., Aguilar-Luzón, M. C., y Salguero de Ugarte, M. F. (2009). Predictive validity of perceived emotional intelligence on nursing students' self-concept. Nurse Education Today, 29, 801-808.

5. Bar-On, R. (2000). Emotional and Social intelligence: Insights from emotional quotient Inventory. En R. Bar-On y J.D. Parker (Eds.), The handbook of emotional intelligence (pp.363-388). San Francisco: JosseyBass.

6. Bar-On, R. (2006). The Bar-On Model of Emotional-Social Intelligence (ESI). Psicothema., 18, 13-25.

7. Bermúdez, J. (1999). Personality and health protective behaviour. European Journal of Personality, 13, 83-103.

8. Botterill, C., y Brown, M. (2002). Emotion and perspective in sport. International Journal of Sport Psychology, 33, 38-60.

9. Carlin, M., y Garcés de los Fayos, E. (2010). El síndrome de Burnout: evolución histórica del concepto del contexto laboral al ámbito deportivo. Anales de Psicología, 26 (1), 169-180.

10. Ciarrochi, J., Forgas, J., y Mayer, J. (2001, 2006). Measuring Emotional Intelligence (25-45). In Ciarrochi, J., Forgas, J. \& Mayer, J. (Eds.) Emotional Intelligence in everyday life: a scientific inquiry. Philadelphia: Psychology Press.

11. Cox, R. H. (2009). Psicología del Deporte. Madrid: Panamericana.

12. De Francisco, C., Garcés de los Fayos, E.J., y Arce, C. (2013). Burnout en deportistas: prevalencia del síndrome a través de dos medidas. Cuadernos de Psicología del deporte, 14 (1), 29-38.

13. DeNeve, K.M., y Cooper, H. (1998). The happy personality: a metaanalysis of 137 personality traits and subjective well-being. Psychological Bulletin, 124 (2), 197-229.

14. Extremera, N., Durán, A., y Rey, L. (2007). Perceived emocional intelligence and dispositional optimism-pessimism: Analyzing their role in predicting psychological adjustment among adolescents. Personlaity and individual Differences, 42, 1069-1079.
15. Extremera, N., Durán, A., \& Rey, L. (2009). The moderating effect of trait meta-mood and perceived stress on life satisfaction. Personality and Individual Differences, 47(2), 116-121.

16. Extremera, N. y Fernández-Berrocal, P. (2004). El papel de la Inteligencia emocional en el alumnado: evidencias empíricas. Revista Electrónica de Inestigación Educativa,6(2). Extraido de http://redie/uabc.mx/ vol6no2/contenido-extremera.html

17. Extremera, N., y Fernández-Berrocal, P. (2005). Perceived emotional intelligence and life satisfaction: Predictive and Incremental validity using the trait Meta-mood Scale. Personality and Individual Differences., 39, 937-948.

18. Extremera, N., y Fernández-Berrocal, P. (2006). Emotional intelligence as predictor of mental, social and physical health in university students. The Spanish Journal of Psychology, 9, 45-51.

19. Extremera, N., Fernández-Berrocal, P., y Salovey, P. (2006) Spanish versión of the Mayer-Salovey-Caruso Emocional Intelligence Test (MSCEIT). Versión 2.0.: Reliabilities, age and gender differences. Psicothema, 18, 42-48.

20. Fernández-Berrocal, P. Alcaide, R., Extremera, N., y Pizarro, D. (2006) The role of emotional intelligence in anxiety and depresión among adolescents. Individual Differences Research, 4, 16-27.

21. Fernández-Berrocal, P., Extremera, N., y Ramos, N. (2004). Validity and reliability of the Spanish modified version of the Trait Meta-Mood Scale. Psychological Reports, 94, 751-755.

22. Fernández-Berrocal, P., y Ruiz, D. (2008). La inteligencia emocional en la educación. Revista Electrónica de Investigación Psicoeducativa., 6(2), 421-436.

23. Furnham, A., y Petrides, K. (2003), Trait Emotional Intelligence and happiness. Social Behaviour and Personality, 31(8), 815-823.

24. Garaigordobil, M. y Oñederra, J. A (2010). Inteligencia emocional en las víctimas de acoso escolar y en los agresores [Emotional intelligence in victims of school bullying and in aggressors]. European Journal of Education and Psychology, 3(2), 243-256.

25. Garcés de Los Fayos, E. J., De Francisco, C y Arce, C. (2012). Inventario de Burnout en Deportistas Revisado (IBD-R). Revista de Psicología del Deporte 21 (2), 271-278.

26. Garcés de Los Fayos, E.J. y Vives Benedicto, L. (2003). Hacia un modelo teórico explicativo de burnout en deportistas: Una propuesta integradora. EduPsykhé, 2(2), 221-242.

27. Goleman, D. (2005). Emotional intelligence. New York: Knopf Pulishing Group.

28. González, J. Garcés de los Fayos, E., y Ortega, E. (2014). Avanzando 
en el camino de diferenciación psicológica del deportista. Ejemplos en diferencias en género y modalidad deportiva. Anuario de Psicología, 44 (1), 31-44.

29. Hansen, D., Larson, R., y Dworkin, J. (2003). What Adolescents Learn in Organized. Youth Activities: A Survey of SelfReported Developmental Experiences. Journal of research on adolescence, 13 (1), 25-55.

30. Hopkins, W. (2005). Competitive Performance of Elite Track-andField Athletes: Variability and Smallest Worthwhile Enhancements. Sportscience, 9, 17-20.

31. Jiménez, M.I. y López-Zafra, E. (2008). El autoconcepto emocional como factor de riesgo emocional en estudiantes universitarios. Boletín de Psicología, 93, 21-39.

32. Jiménez, M.I. y Lopez Zafra, E. (2011) Actitudes sociales y adaptación social en adolescentes espańoles: el papel de la inteligencia emocional percibida. Revista de Psicologia Social, 26(1), 105-117.

33. Lane, A. M., Devonport, T. J., Soos, I., Karsai, I., Leibinger, E., y Hamar, P. (2010). Emotional intelligence and emotions associated with optimal and dysfunctional athletic performance. Journal of sports science \& medicine, 9 (3), 388-392.

34. Lane, A. M., Thelwell, R., Devonport, T. (2009). Emotional Intelligence and Mood States associated with Optimal Perfromance. E-Journal of Applied Psychology, 5 (1), 67-73.

35. Martins, A., Ramalho, N., y Morin, E. (2010). A comprehensive metaanalysis of the relationship between Emotional Intelligence and health. Personality and Individual Differences 49, 554-564.

36. Meyer, B. B., Fletcher, T. B. (2007). Emotional intelligence: A theoretical overview and implications for research and professional practice in sport psychology. Journal of Applied Sport Psychology, 19, 1-15.

37. Mayer, J. D., Roberts, R. D., Barsade, S. G. (2008). Human abilities: Emotional Intelligence. Annual Review of Psychology, 59, 507-536.

38. Mayer, P. y Salovey, P. (2007). ¿Qué es la Inteligencia emocional? En J. M. Maestre y P. Fernández-Berrocal (Coords), Manual de Inteligencia emocional, (pp. 25-45). Madrid: Pirámide.

39. Mayer, J. D., Salovey, P., y Caruso, D. (2000). Models of emotional intelligence. En R.J. Sternberg (ed.), Handbook on intelligence (pp. 396420). New York: Cambrigde.

40. Mikolajczak, M., Luminet, O., Menil, C. (2006). Predicting resistance to stress: Incremental validity of trait emotional intelligence over alexithymia and optimism. Psicothema., (18), 79-88.

41. Mikolajczak, M., Nelis, D., Hansenne, M., y Quoidbach, J. (2008). If you can sadness, yoy can probably regulate shame: Associations between trait emotional intelligence, emotion regulation and coping efficiency across discrete emotions. Personality and Individual Differences, 44, 1356-1368.

42. Mora, J. A., y Martín, M. L. (2007). Inteligencia Emocional y personalidad. En Libro de resúmenes del I Congreso Internacional de Inteligencia emocional (p.163). Málaga, España.

43. Moreno, B., Rodríguez, A., Garrosa, E., Morante, M.E. (2005). Antecedentes organizacionales del acoso psicológico en el trabajo: un estudio exploratorio. Psicothema, 17 (4), 648-653.

44. Newsome, S., Day, A., y Catano, V. (2000). Assessing the predictive validity of emotional intelligence. Personality and Individual Differences, 29, 1005-1016.

45. Palomera, R., Fernández-Berrocal, P., y Brackett, M. (2008). La inteligencia emocional como una competencia básica en la formación inicial de los docentes: algunas evidencias. Revista Electrónica de Investigación Psicoeducativa, 6, (2), 437-454.
46. Parker, J., Summerfeldt, L., Hogan, M., y Majeski, S. (2004). Emotional Intelligence and academic success: examinig the transition from high school to university. Personality and Individual Differences, 36 (1), 163-172.

47. Paton C.D., y Hopkins W.G. (2005).Competitive performance of elite Olympic-distance triathletes: reliability and smallest worthwhile enhancement. Sportscience, 9, 1-5.

48. Pena, M., y Repetto, E. (2008). Estado de la investigación en Espańa sobre Inteligencia Emocional en el ámbito educativo. Revista Electrónica de Investigación Psicoeducativa, 6(2), 400-420.

49. Pérez, N., y Castejón, J. (2007). La Inteligencia emocional como predictor del rendimiento académico en estudiantes universitarios. Ansiedad y estrés, 13 (1), 119-129.

50. Petrides, K., Frederickson, N., y Furham, A. (2004). The role of trait emotional intelligence in academic performance and deviant behavior at school. Personality and Individual Differences 36 (2), 277-293.

51. Petrides, K. y Furnham, A. (2001). Trait emotional intelligence: psychometric investigation with referente to established trait taxonomies. European Journal of Personality, 15, 425-448.

52. Ramos, N., Fernández-Berrocal, P., Extremera, N (2007). Perceived emotional intelligence facilitates cognitive-emotional processes of adaptation to an acute stressor. Cognition and Emotion, 21 (4), 758-772.

53. Reiff, H., Hatzes, N., Bramel, M., y Gibbon, T. (2001). The relation of LD and gender with emotional intelligence in college students. Journal of Learning Disabilities, 34 (1), 66-78.

54. Ros, A., Moya, F., y Garcés de los Fayos, E (2013). Inteligencia emocional y deporte: situación actual del estado de la investigación. Cuadernos de Psicología del Deporte, 13 (1), 105-112.

55. Salguero, J. M., Iruarrizaga I. (2006). Relationship between perceived emotional intelligence and negative emotionality: Anxiety, anger and sadness/depression. Ansiedad y Estrés, 12(2-3), 207-221.

56. Salguero, J. M., Palomera, R., y Fernández- Berrocal, P. (2012). Perceived emotional intelligence as predictor of psychological adjustment in adolescents: a 1-year prospective study. European Journal of Psychological Education, 27 (1), 21-34.

57. Salovey, P., y Grewal, D. (2005).The Science of emotional intelligence. Currents Directions in Psychological Science, 14, 281-285.

58. Salovey, P., Stroud, L., Woolery, A., y Epel, E. (2002). Perceived emotional intelligence, stress reactivity, and symptoms reports: further explorations using the Trait Meta-Mood Scale. Psychology annd Health, 17, 611-627.

59. Sanz J., Silva F., y Avia, M. D. (1999). La evaluación de Personalidad desde el modelo de los "Cinco Grades": El Inventario de Cinco- Factores NEO (NEO-FFI) de Costa y McGrae. En: F. Silva, (dir.) Avances en Evaluación Psicológica. Valencia: Promolibro.

60. Smith, E., y Christensen, D. S. (1995). Psychology skills as predictor of performance and survivalin professional baseball. Journal of Sport \& Exercise Psychology, 17, 399-415.

61. Van Heck, G.L. (1997). Personality and physical health: Toward an ecological approach to health-related personality research. European Journal of Personality, 11, 415-443.

62. Zeidner, M., Roberts, R., y Matthews, G. (2008). The Science of Emotional Intelligence: current consensus and controversies. European Psychologist., 13(1), 64-78. 